\title{
Performance Analysis of Cluster-based Wireless Sensor Networks with Application Constraints
}

\author{
Abdul Sattar Malik, Jingming Kuang, Jiakang Liu, Wang Chong \\ School of Information and Electronics, Beijing Institute of Technology, Beijing-100081, P. R. China \\ Maliksattar777@gmail.com, jmkuang@public3.bta.net.cn, liujk@bit.edu.cn, wangchong@bit.edu.cn
}

\begin{abstract}
Clustering is an efficient techniques used to achieve the specific performance requirements of large scale wireless sensor networks. In this paper we have carried out the performance analysis of cluster-based wireless sensor networks for different communication patterns formed due to application constraints based upon LEACH protocol, which is among the most popular clustering protocols proposed for these types of networks. Simulation results based upon this protocol identify some important factors that induce unbalanced energy consumption among sensor nodes and hence affect the network lifetime. This highlights the need for an adaptive clustering protocol that can increase the network lifetime by further balancing the energy consumption among sensor nodes. Paper concludes with some recommendations for such protocol.
\end{abstract}

Index Terms - wireless sensor networks, clustering protocols, communication patterns, energy efficiency, network lifetime

\section{INTRODUCTION}

Advances in wireless communications and electronics have fostered the development of relatively in-expensive and low-power wireless sensor nodes that are extremely small in size and communicate un-tethered in short distances. Wireless sensor node is a battery-operated device, capable of sensing physical quantities, data storage, limited amount of computational and signal processing capability and wireless communication. A Wireless Sensor Network (WSN) is composed of a large number of sensor nodes that are densely deployed either inside the phenomenon or very close to it [1]. WSN can have one or more sinks or Base Stations (BS) which collect data from sensor nodes. These sinks act as an interface through which the WSN interacts with the outside world. WSNs facilitate monitoring and controlling of physical environments from remote locations with better accuracy. Sensor nodes in WSNs have various energy and computational constraints because of their inexpensive nature and ad-hoc method of deployment. They usually employ performance metrics that are different from those of conventional networks, emphasizing on low power consumption and low cost rather than data throughput or channel efficiency. Major advantages of WSNs over the conventional networks deployed for the same purpose are greater coverage, accuracy, reliability and all of the above at a possibly

Manuscript received January 18, 2009; revised June 13, 2009; accepted July 22, 2009. lower cost. Some of the early works on WSNs have discussed these benefits in detail [2], [3], [4].

WSNs have innumerable number of applications in various fields triggering a great deal of research attention in recent times. Several applications have already been envisioned for these types of networks ranging form environment, agriculture, industry, object detection, its classification and tracking, disaster relief, facility management, preventive maintenance, medicine and health to name the few [5], [6], [7], [8]. WSN applications can be classified according to the nature of the functionalities, and in addition according to the data delivery and communication pattern between the sensor nodes and the base station [9]. According to their functionalities, WSN applications can be classified into four main categories: monitoring applications, tracking applications, controlling applications, and hybrid applications.

Based on the data delivery and communication pattern between the sensors nodes and the base station (sink), WSN applications can be classified into four classes: The continuous model on which sensor nodes periodically report to the observer about a physical parameter. For example, nodes in a network monitoring precision agriculture are periodically sending temperature, light levels, and soil moisture to the observer over a multi-hop network. The event-driven model where sensors send information only if an event of interest is detected. For example, sensors that are deployed to detect forest fires will report only when the temperature exceeds a certain degree. The observer-initiated model on which the observer may be interested only in the occurrence of an event at certain geographic area. In this case, the observer will send an explicit request to specific sensors. Note that such query can be predefined in advance by the application to be answered by specific sensors at certain intervals. This can be viewed as a prescheduled observerinitiated model. The hybrid model where some or all of the above three models coexist in the same network.

Clustering is one of the efficient techniques used to achieve the specific performance requirements of WSNs. Specially, in large scale networks, consisting of hundreds or even thousands of nodes, clustering techniques can enable scalability, resource allocation, data aggregation, and energy conservation. At the same time, on top of a clustering architecture, different schemes can be employed at different layers for example MAC protocols, routing protocols, resources allocation schemes, duty 
cycle assignment and scheduling schemes, data aggregation and signal processing algorithms. Clustering is the process of dividing a network into groups of sensor nodes called clusters. Each cluster is managed by a chosen Cluster-Head $(\mathrm{CH})$. CHs are either selected by the local base station using a centralized algorithm or they contend for this using a distributed algorithm and then ordinary nodes join these CHs based upon cluster joining algorithm. After the completion of the clustering phase, CHs collect \& aggregate data from their members and report aggregated data to the sink. Role of CHs can be rotated to share the burden and balance the energy consumption among sensor nodes.

Although formation and maintenance of clusters introduces additional cost due to the control messages required for the purpose, still cluster-based WSNs have taken much attention of the researchers due to their better performance. Distributed, dynamic and randomized clustering schemes are interesting due to their simplicity, feasibility, and effectiveness in providing energy-efficient utilization, load balancing and scalability simultaneously [10]. Being a dominant representative in this class, LEACH (Low Energy Adaptive Clustering Hierarchy) [11], [12] has attracted intensive attention and become a well studied and popularly referred baseline since its appearance. Much work [13], [14], [15], [16] has been carried out to enhance LEACH.

In order to evaluate the performance of the LEACH protocol, the authors used a communication model where nodes always have data to send to the end user and nodes located close to each other have correlated data. In other words the authors used an event-driven model where the event was occurring always and every where all around the sensor field. Simulation results presented in [11] do indicate the better performance of the LEACH protocol over static clustering and MTE (Minimum Transmission Energy) protocol [17], [18] for such communication patterns. In order to further optimize the performance of the LEACH protocol, their remains a need to evaluate the performance of the LEACH protocol for different communication patterns formed due to the application constraints for WSNs.

In this paper we have carried out the performance analysis of cluster-based WSNs based upon LEACH protocol for different communication patterns between the sensor nodes and the base station due to application constraints. The main focus is to identify the factors that affect the network lifetime by inducing unbalanced energy consumption among sensor nodes for different communication patterns due to application constraints. The remainder of the paper is arranged as follows. Section-II provides the background about cluster-based WSNs based upon LEACH protocol. The details of the proposed scenarios based upon the different communication patterns formed due to application constraints that were chosen for the performance evaluation by developing a simulation environment has been discussed in section-III. Methodology for the development of the simulation environment using OPNET to identify the factors inducing unbalanced energy consumption among sensor nodes and thus affecting the network lifetime has been discussed in section-IV. Based upon the simulation results, conclusions have been drawn and some recommendations for future work have been proposed in section-V.

\section{BRIEF REVIEW OF LEACH}

LEACH [11], [12] is a distributed clustering protocol that utilizes randomized rotation of local CHs to evenly distribute energy consumption among sensor nodes in the network. In LEACH, the whole operation is divided into many rounds. Every round includes a set-up phase followed by a steady-state phase. During the set-up phase, nodes are organized into clusters with each cluster having its own $\mathrm{CH}$ through short message communications using Carrier Sense Multiple Access (CSMA) MAC protocol. Each $\mathrm{CH}$ sets up TDMA schedules for its member nodes which are later used to exchange data between the member nodes and the $\mathrm{CH}$ during steady-state phase. The steady state phase consists of many frames. Since the duration of each slot in which a node transmits data is constant, so the time to send a frame of data depends on the number of nodes in the cluster. Data are transferred from member nodes to $\mathrm{CHs}$ according to the assigned TDMA schedules during each frame, aggregated to reduce redundant data and then passed on to the BS. Fig. 1 shows the round timeline diagram for the LEACH protocol. To reduce inter-cluster interference, members of each cluster communicate using Direct-Sequence Spread Spectrum (DSSS).

LEACH forms clusters by using a distributed algorithm, where nodes make autonomous decisions without any centralized control. Each node elects itself to be a $\mathrm{CH}$ at the beginning of round $r$ with probability $P_{i}(t)$ such that the expected number of $\mathrm{CHs}$ for that round is $k$. Thus, if there are $N$ nodes in the network

$$
E(C H)=\sum_{i=1}^{N} P_{i}(t) * 1=k
$$

To ensure that all nodes are $\mathrm{CHs}$ the same number of time, LEACH protocol requires each node to be a $\mathrm{CH}$ once in $N / k$ rounds on average. If $C_{i}(t)$ is the indicator function determining whether or not node has been a $\mathrm{CH}$ in the most recent $(r \bmod N / k)$ rounds (i.e. $C_{i}(t)=0$ if node has been a $\mathrm{CH}$ and one otherwise), then each node should choose to become a $\mathrm{CH}$ at round $r$ with probability

$$
P_{i}(t)= \begin{cases}\frac{k}{N-k^{*}(r \bmod N / k)}: & C_{i}(t)=1 \\ 0 \quad: & C_{i}(t)=0\end{cases}
$$

\section{A. Radio Energy Dissipation Model}

For simplicity LEACH proposes a simple model for the radio hardware energy dissipation where the transmitter dissipates energy to run the radio electronics and the power amplifier, and the receiver dissipates energy to run the radio electronics, as shown in Fig. 2. For the analytical and simulation work described in this paper, both the free space $\left(d^{2}\right.$ power loss) and the 
multipath fading ( $d^{4}$ power loss) channel models were used, depending on the distance between the transmitter

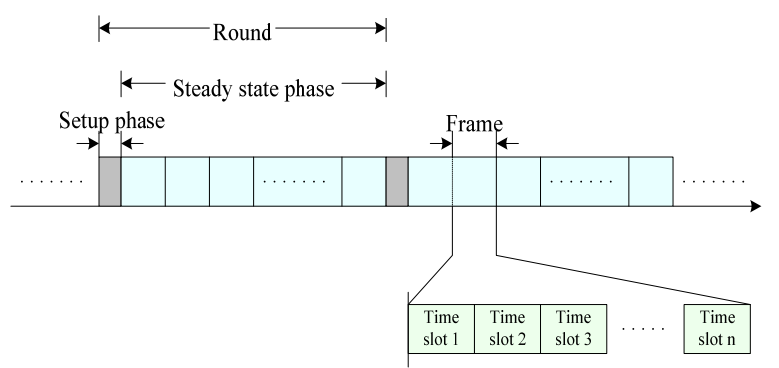

Figure 1: Round timeline diagram for LEACH protocol

and receiver. Power control can be used to invert this loss by appropriately setting the power amplifier. If the distance is less than a threshold, the free space model is used; otherwise, the multipath model is used. Thus, to transmit an l-bit message a distance $d$, the radio expends

$$
\begin{aligned}
E_{T x}(l, d) & =E_{T x \_ \text {elect }(l)+E_{T x_{-}} \operatorname{amp}(l, d)} \\
& = \begin{cases}l E_{\text {elect }}+l \varepsilon_{f s} d^{2} & d<d_{0} \\
l E_{\text {elect }}+l \varepsilon_{m p} d^{4} & d \geq d_{0}\end{cases}
\end{aligned}
$$

and to receive this message, the radio expends

$$
\begin{aligned}
E_{R x}(l) & =E_{R x}-\operatorname{elect}(l) \\
& =l E_{\text {elect }}
\end{aligned}
$$

Table 1 summarizes different communication energy parameters as proposed in [11] used in the simulation work described in this paper.

\section{B. Traffic Patterns of the LEACH Protocol}

As previously mentioned, in order to evaluate the performance of the LEACH protocol, the authors used a communication model where nodes always have data to send to the end user and nodes located close to each other have correlated data. In other words the authors used an event-driven model where the event was occurring always and every where all around the sensor field. So in order to make effective use of bandwidth resources $\mathrm{CHs}$ in any round divide the round time into frames where frame duration and hence the number of frames per round for each cluster depends upon the number of member nodes in that cluster, i.e. Small clusters will have more frames per round and vice versa. Such traffic patterns cover only a limited number of scenarios for WSN applications. Considering the classification of WSN applications based upon the data delivery \& communication pattern between the sensor nodes and the base-station, there can be wide range of scenarios following the continuous model, event driven model, observer initiated model and hybrid model.

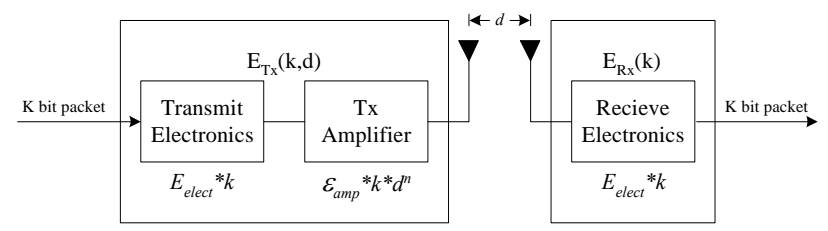

Figure 2: Radio energy dissipation model
TABLE I. RADIO MODEL COMMUNICATION PARAMETERS

\begin{tabular}{c|c}
\hline Parameter name & Value \\
\hline RF Radio circuitry energy $\left(E_{\text {elect }}\right)$ & $50 \mathrm{~nJ} / \mathrm{bit}$ \\
\hline Amplifier energy for Free space loss $\left(\varepsilon_{f s}\right)$ & $10 \mathrm{pJ} / \mathrm{bit} / \mathrm{m}^{2}$ \\
\hline Amplifier energy for Multipath loss $\left(\varepsilon_{m p}\right)$ & $0.0013 \mathrm{pJ} / \mathrm{bit} / \mathrm{m}^{4}$ \\
\hline Threshold distance $\left(d_{0}\right)$ & $87 \mathrm{~m}$ \\
\hline Data aggregation energy $\left(E_{D A}\right.$ & $5 \mathrm{~nJ} / \mathrm{bit} / \mathrm{signal}$ \\
\hline
\end{tabular}

\section{Details of the Proposed Scenarios}

The lifetime of a WSN can be defined as the time elapsed until the first node dies, the last node dies, or a fraction of nodes die [4], [19]. In a WSN, in addition to minimizing energy expenditure, a protocol design should also take fairness into consideration to achieve a sharp edge effect [19], i.e. individual nodes drain out of energy at similar time. Thus when the network loses its functionality, remaining active nodes should have little residual energy. An ideal scheme should enable the network to operate for the longest possible time, while each node burns its energy at the same pace. Our objective is to determine how much extent LEACH

protocol achieves sharp edge effect and what are those factors that induce unbalanced energy consumption among sensor nodes and hence affect the network lifetime.

For the simulation work described in this paper, we used a 100-node network with the same network specifications as were used in [11]. The bandwidth of the channel was set to $1 \mathrm{Mb} / \mathrm{s}$, each data message was 500 bytes long, and the packet header for each type of packet was 25 bytes long. Table II summarizes different network parameters.

From energy consumption perspective, as per LEACH specifications, each $\mathrm{CH}$ node dissipates energy receiving signals from its member nodes, aggregating the signals and transmitting the aggregated signal to the BS. Assuming that the BS is far from the sensing area, the energy dissipation follows the multipath model $\left(d^{4}\right.$ power loss). So the energy dissipated by the $\mathrm{CH}\left(E_{C H}\right)$ during a single frame is

$$
E_{C H}=l E_{\text {elect }}\left(\frac{N}{k}-1\right)+l E_{D A} \frac{N}{k}+l E_{\text {elect }}+l \varepsilon_{m p} d_{t o B S}^{4}
$$

$(N / k-1)$ is the average number of non-CH nodes in each cluster with $k$ clusters in each round.

Each non-CH or member node only needs to transmit its data to the $\mathrm{CH}$ once during each frame. Presumably the distance to the $\mathrm{CH}$ is small, so the energy dissipation follows the free-space model ( $d^{2}$ power loss). Thus, the energy dissipated in each non- $\mathrm{CH}$ node $\left(E_{\text {non- } \mathrm{CH}}\right)$ during a frame is

$$
E_{\text {non-CH }}=l E_{\text {elect }}+l \varepsilon_{f s} \frac{1}{2 \pi} \frac{M^{2}}{k}
$$


$M^{2} / 2 \pi k$ is the average squared distance of the non- $\mathrm{CH}$ nodes from their $\mathrm{CH}$.

TABLE II. SENSOR NETWORK PARAMETERS

\begin{tabular}{c|c}
\hline Parameter & Value \\
\hline Sensing area $(M)$ & $100 \times 100$ \\
\hline Number of sensor nodes $(N)$ & 100 \\
\hline$D_{\text {toBS }}($ min $)$ & 75 \\
\hline$D_{\text {toBS }}($ max $)$ & 185 \\
\hline Initial node energy level $\left(E_{\text {init }}\right)$ & $2.0 \mathrm{~J}$ \\
\hline Data packet size $(l)$ & $4000 \mathrm{bits}$ \\
\hline
\end{tabular}

For the simulation work described in this paper, we used event driven communication model and continuous communication model. Following are the details of these communication models.

\section{A. Event Driven Model}

In order to evaluate the performance of the LEACH protocol, the authors used a communication model where nodes always have data to send to the end user and nodes located close to each other have correlated data. This corresponds to an event-driven model where the event was occurring always and every where all around the Sensor Field (SF). In general there can be scenarios where the Event Field (EF) is confined within a certain region in the SF. For example sensor nodes are deployed to detect the forest fire but the fire is confined within a certain region of the overall SF. Fig. 3 describes those kinds of scenarios.

For all those scenarios in which the EF is confined within a certain region of the SF, only those member nodes which are located inside the EF have data to send to their CHs. In general this gives rise to three possible scenarios.

Cluster fully confined within the Event Field: In this case, since all the members of a cluster are located inside the EF so all the non-CH nodes will detect that event and then transmit this information to the BS and dissipate energy for this transmission activity. $\mathrm{CH}$ nodes will dissipate energy while receiving signals from member nodes, aggregating those signals and then communicating the aggregated data to the BS as given in equation 4 during each frame.

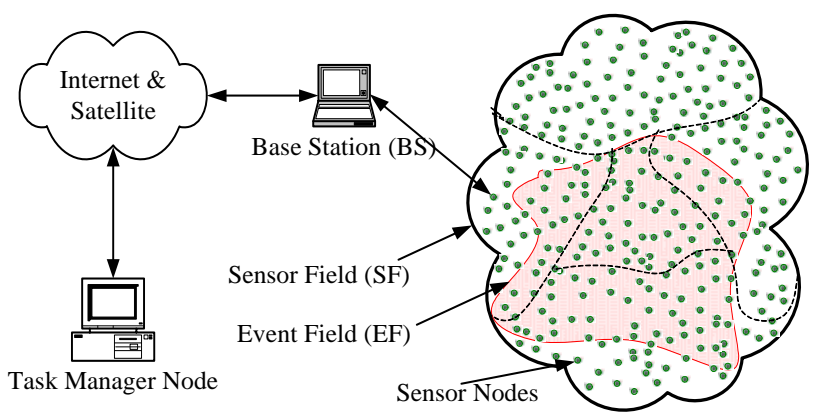

Figure 3: A WSN scenario where the event field is confined within a certain region of the sensor field
Cluster located outside the Event Field: In this case, all the members of a cluster are located outside the EF. Since all the non-CH nodes will not detect any event so they will not have any information to transmit to the $\mathrm{CH}$ and hence all these nodes will not dissipate any energy in this aspect. On the other hand the $\mathrm{CH}$ node has to keep its receiver on for any possible communication from its member nodes so it will be consuming reception part of the energy. During its own time slot for data transmission to the BS, since is does not have any data to aggregate and report to the BS so it will not be consuming any energy due to data aggregation and BS transmission.

Cluster partially confined within the Event Field: In this case, some of members of the cluster are located inside the EF while the others are located outside the EF. Only those non- $\mathrm{CH}$ nodes which are located inside the EF will detect that event and then transmit this information to their $\mathrm{CH}$ and dissipate energy for this transmission activity. Those non-CH nodes which are located outside the EF, will not detect any event so they will not have any information to transmit to their $\mathrm{CH}$ and hence these nodes will not dissipate energy in this regard. The CH node has to keep its receiver on for any possible communication whether it is there or not so the reception part of the energy will be there. During its own time slot for data transmission to the BS, it has to aggregate the only that information which it received from those member nodes which were located inside the EF along with its own information if it is also located inside the EF and has to transmit the aggregated information to the BS.

We define E/S as the ratio of the EF area to the SF area. For example $\mathrm{E} / \mathrm{S}=0.2$ means $\mathrm{EF}$ is confined only to the $20 \%$ of the total SF. For the sake of simplicity if we assume that the EF is confined in the form of a circle from the centre of the SF and if A is the total area of the $\mathrm{SF}$, then for a particular value of the $\mathrm{E} / \mathrm{S}$, we can calculate the radius of the $\mathrm{EF}\left(\mathrm{R}_{\mathrm{EF}}\right)$ as follows

$$
R_{E F}=\sqrt{\frac{A \times \frac{E}{S}}{\pi}}
$$

Using different values of $\mathrm{E} / \mathrm{S}$, we can determine the corresponding radius of the EF. Nodes located within the circle will be able to detect the event and report the sensed value to their $\mathrm{CH}$.

\section{B. Continous Model}

For a broader range of WSN applications sensor nodes can be tasked with periodic reporting of the measured values. The reporting period for the measured values is an application dependent parameter. Number of frames per round will not vary with the cluster size as was the case with an event driven model. Under the ideal circumstances, the number of frames value should be set such that each node should act as $\mathrm{CH}$ only once during the network lifetime. In order to have a detailed analysis of the LEACH protocol for the continuous model, we set three different values of number of frames per round such that each node should have $\mathrm{CH}$ role assignment once, two times and four times respectively. 


\section{SiMULATION WORK}

Based upon the specifications of the LEACH protocol from section II and objectives for the proposed work defined in section III, there is a need to carry out the performance analysis of the LEACH protocol for different communication patterns formed due to application constraints. OPNET was chosen as the simulation platform. This tool is a discrete-event simulator developed by MIL3 Corporation and it provides a comprehensive development environment for the specification, simulation and performance analysis of communication networks. As a building block, a sensor node model was developed. The base scenario of the simulation environment comprised of 100 randomly distributed nodes in a sensing area of $100 \times 100 \mathrm{~m}^{2}$ and a BS located outside the sensing area as per network specifications listed in table II.

Fig. 4 (a) and (b) show the total number of effective data signals received at the BS over time and total effective data received at the BS for a given amount of energy respectively for different values of E/S. For smaller values of $\mathrm{E} / \mathrm{S}$, only nodes present inside the $\mathrm{EF}$ reported the information to their corresponding $\mathrm{CH}$ which transferred this information to the BS. For all those clusters in which all the members are located outside the $\mathrm{EF}$, even though the non- $\mathrm{CH}$ are not dissipating any energy for transmission as they don't detect any event but

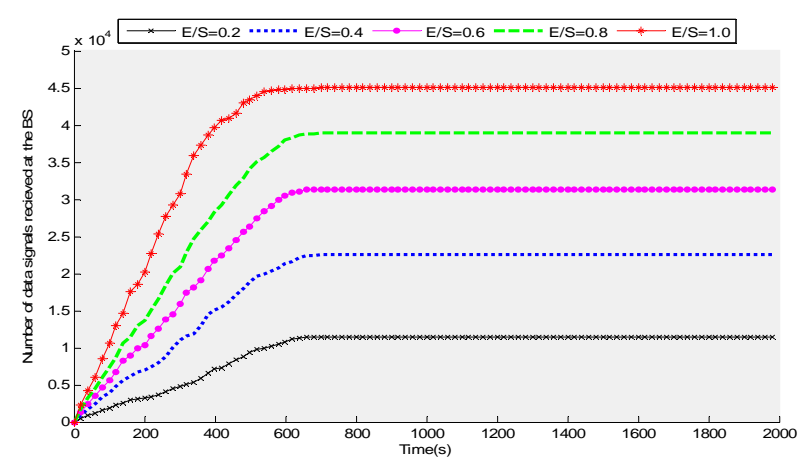

(a) still their corresponding $\mathrm{CH}$ has to dissipate energy as it has to keep its receiver on for the possible communication from its member nodes, but at the end of the frame as the $\mathrm{CH}$ node don't have any data to transmit to the BS so the $\mathrm{CH}$ node will neither dissipate energy for data aggregation nor for data transmission. On the other hand for all those scenarios, in which clusters are partially confined within the EF, only those member nodes present inside the EF will dissipate energy while transmitting their data to their $\mathrm{CH}$ and the $\mathrm{CH}$ node whether it is located inside or outside the EF, has to aggregate the data which it has received form those limited number of nodes and has to report that data to the $\mathrm{BS}$, so the $\mathrm{CH}$ node will be consuming approximately the same amount of energy as would have been the base in which the whole cluster is confined within the EF.

Fig. 5 (a) shows the total number of nodes that remain alive over the simulation time for different values of E/S. In Fig. 5 (b) we have plotted the total number of nodes that remain alive per amount of data received at the BS for different values of E/S. Again for smaller values of $\mathrm{E} / \mathrm{S}$, since with the passage of time, nodes located inside the EF run out of their energy, so the only nodes that remain alive are the nodes which are located outside the EF which consume energy while idle listening. So the rate at which the nodes run out of their energy decreases as the simulation time increases.

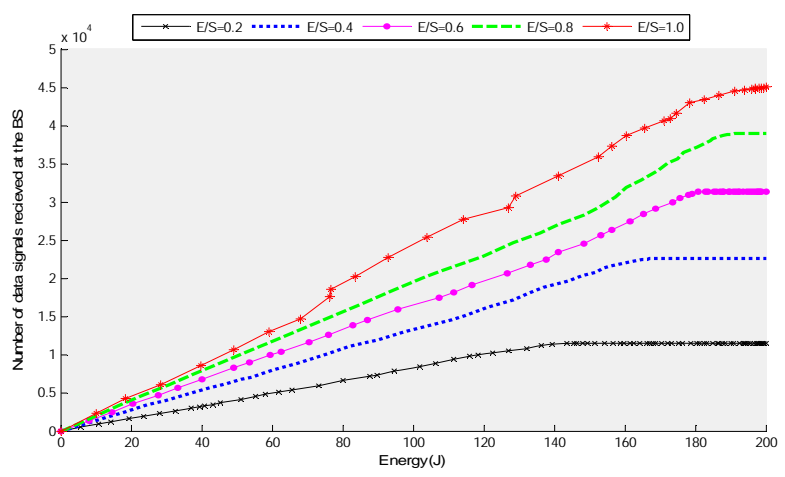

(b)

Figure 4: (a) Number of data signals received at the BS over time for different values of E/S (the ratio of event field area versus sensor field area) (b) Number of data signals received at the BS per given amount of energy for different values of E/S (the ratio of event field area versus sensor field area

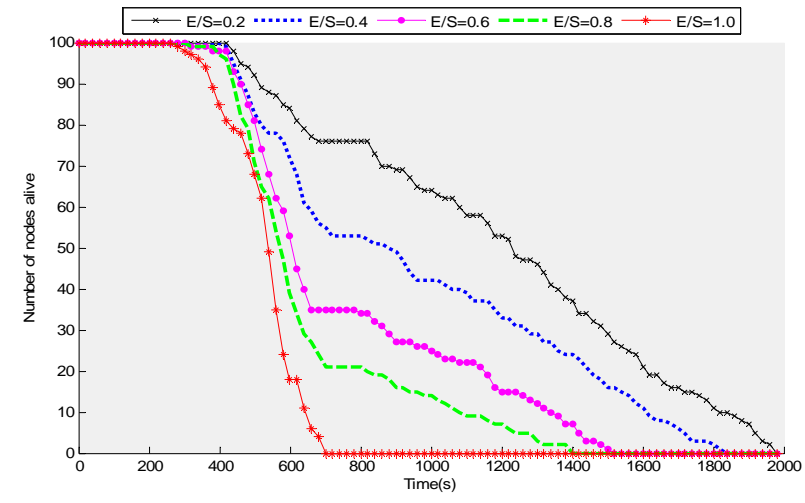

(a)

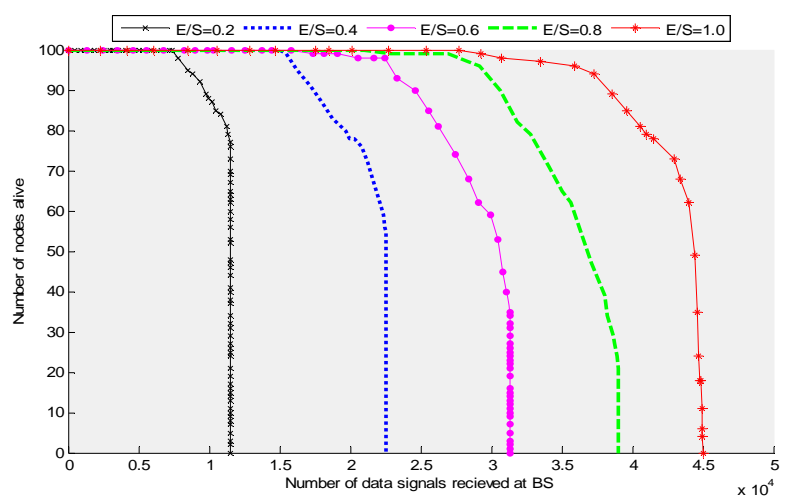

(b)

Figure 5: (a) Number of nodes alive over time for different values of E/S (the ratio of event field area versus sensor field area) (b) Number of nodes alive per amount of data sent to the BS for different values of E/S (the ratio of event field area versus sensor field area) 


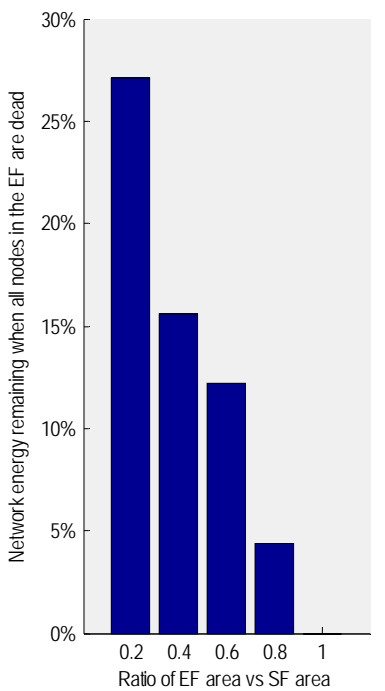

(a)

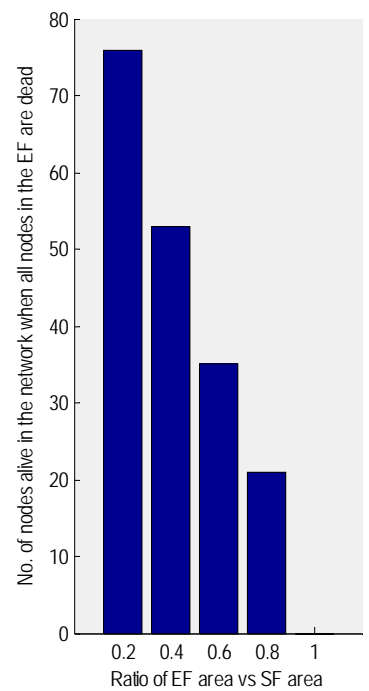

(b)

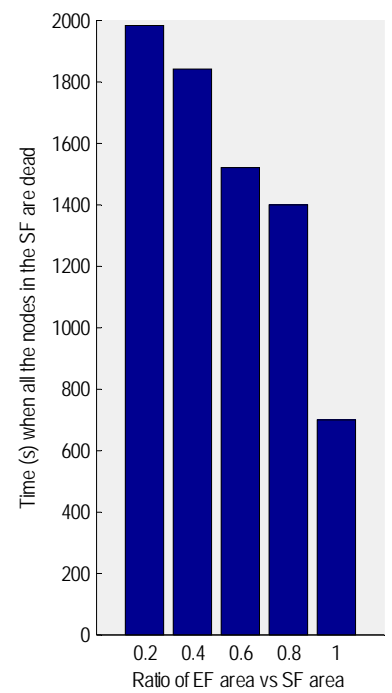

(c)

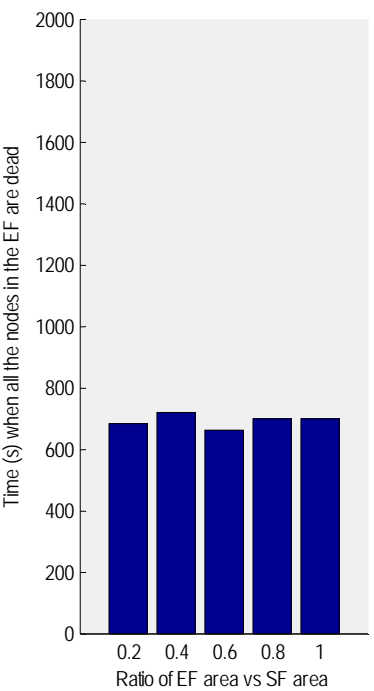

(d)

Figure 6: (a) Network energy remaining when all the nodes in the Event Field (EF) are dead for different values of E/S (the ratio of event field area versus sensor field area) (b) Number of nodes alive in the network when all the nodes in the Event Field (EF) are dead for different values of E/S (the ratio of event field area versus sensor field area)(c) Time (s) when all the nodes in the Sensor Field (SF) are dead for different values of E/S (the ratio of event field area versus sensor field area) (d) Time (s) when all the nodes in the Event Field (EF) are dead for different values of E/S (the ratio of event field area versus sensor field area)

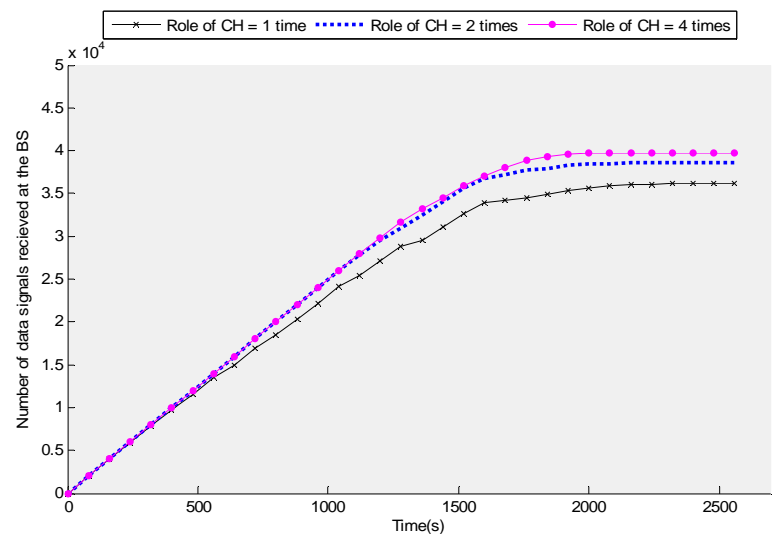

(a)

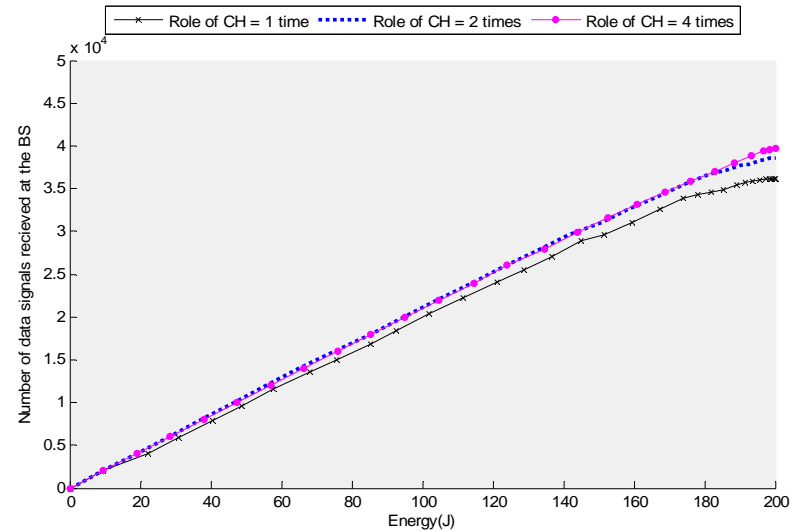

(b)

Figure 7: (a) Number of data signals received at the BS over time for different values of role of CH (b) Number of data signals received at the BS per given amount of energy for different values of role of $\mathrm{CH}$

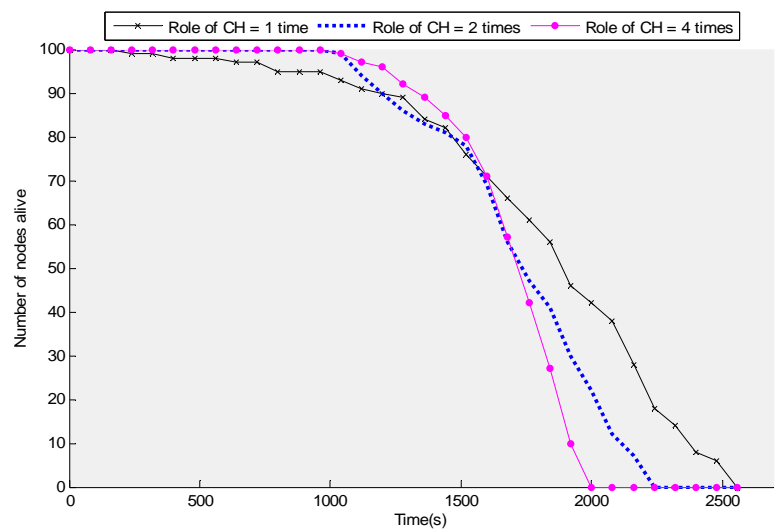

(a)

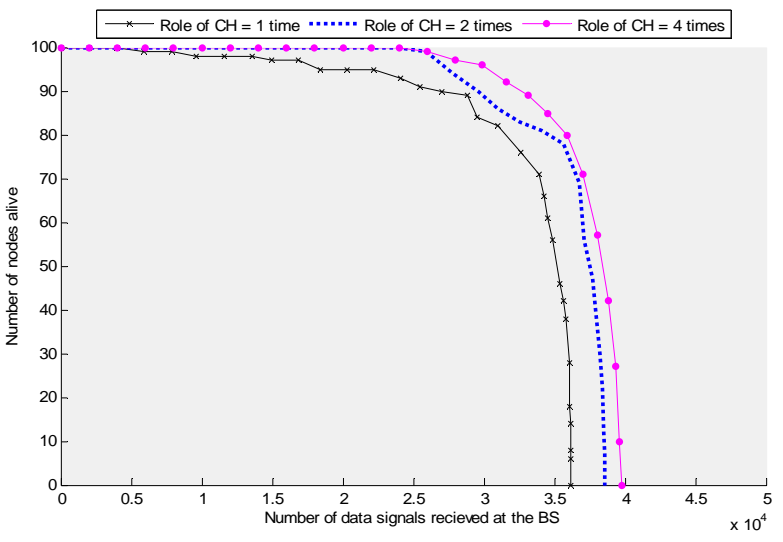

(b)

Figure 8: (a) Number of nodes alive over time for different values of role of $\mathrm{CH}$ (b) Number of nodes alive per amount of data sent to the BS for different values of role of $\mathrm{CH}$ 
Fig. 6 (a) shows the network energy remaining when all the nodes in the EF are dead for different values of E/S. This is the amount of energy for which not even a single communication activity will take place as all nodes that are alive are located outside the EF. Fig. 6 (b) shows the number of nodes alive in the network when all the nodes in the EF are dead for different values of E/S. As it was expected for smaller values of $\mathrm{E} / \mathrm{S}$, the number of nodes that are still alive is much higher as majority of the nodes are located outside the EF.

Fig. 6 (c) shows time in seconds when all the nodes in the SF are dead for different values of E/S. Looking at the graph of Fig. 6(c), initially it looks like that for smaller values of $\mathrm{E} / \mathrm{S}$, the overall network life time is increased. Fig. 6 (d) shows the time in seconds when all the nodes in the EF are dead for different values of E/S. This will be time from which onwards their will be no communication activity that will take place as all those nodes which can detect the event from within the EF are already dead. Interestingly the time value is same approximately for all the values of E/S. So if we define the network lifetime as time when all the nodes within the EF are dead (which is a more realistic definition) then using the LEACH protocol, we are not having an additional lifetime for smaller values of E/S even though still we have a lot of nodes that are still alive.

Fig. 7 (a) and (b) show the total number of effective data signals received at the BS over time and total effective data received at the BS for a given amount of energy respectively for different values of role of $\mathrm{CH}$ during the network lifetime. It is worth mentioning that more frequent repetition of the role of $\mathrm{CH}$ will induce an increased overhead that will be required due to control signal required for the formation of clusters during the cluster setup phase. Ideally a good clustering protocol should provide desired performance when role of $\mathrm{CH}$ value is set to one i.e. each sensor node is set to take the responsibility only once during the network lifetime. Fig. 7 (a) and (b) show that for smaller values of role of $\mathrm{CH}$, lesser amount of data signals are received at the base

\section{REFERENCES}

[1] I. F. Akyildiz, W. Su, Y. Sankarasubramaniam and E. Cayirci, "A survey on sensor networks," IEEE Communications Magazine, vol. 40, no. 8, pp. 102-114, 2002.

[2] Chandrakasan, et al., "Design considerations for distributed micro-sensor systems,” Proc. IEEE 1999 Custom Integrated Circuits Conference (CICC'99), San Diego, CA, USA, May 1999, pp. 279-286.

[3] D. Estrin, L. Girod, G. Pottie, and M. Srivastava, "Instrumenting the world with wireless sensor networks," Proc. IEEE International Conference on Acoustics, Speech and Signal Processing (ICASSP 2001), Salt Lake City, Utah, USA, May 2001, vol. 4, pp. 2033-2036.

[4] D. Estrin, R. Govindan, J. Heidemann, and S. Kumar, "Next century challenges: Scalable coordination in sensor networks," Proc. 5 ${ }^{\text {th }}$ Annual ACM/IEEE International Conference on Mobile Computing and Networking station over time and per given amount of energy. Fig. 8 (a) show the number of nodes alive over time for different values of role of $\mathrm{CH}$. It can be observed that that sharp edge effect is only achieved by higher values of role of $\mathrm{CH}$.

\section{CONCLUSIONS \& RECOMMENDATIONS}

In this paper we have carried out the performance analysis of cluster-based WSNs for different communication patterns formed from the application constraints. Our special focus was on event driven communication models and continuous communication models. For event driven communication modes, along with other parameters, we analyzed the network performance in terms of the number of data signals received at the base station over time, number of data signals received at the BS per unit amount of energy, time during which all the nodes in the SF are dead and the time during which all the nodes in the EF are dead for different values of E/S. It was observed that when we decrease the value of $\mathrm{E} / \mathrm{S}$, the corresponding number of data signals received at the BS also decrease and the time when all the nodes in the SF are dead increases. On the other hand the time during which all the nodes within the EF are dead remains approximately the same for different values of $\mathrm{E} / \mathrm{S}$ and hence after this time even though there are still many nodes which are alive, but since all these nodes are located outside the EF, so they dissipate all of their remaining energy in idle listening whenever they take the role to act as $\mathrm{CH}$. For continuous communication model, we observed that for smaller values of role of $\mathrm{CH}$, the number of nodes alive over time does not follow the sharp edge effect, indicating unbalanced energy consumption among sensor nodes. This highlights the need for a generalized, standardized \& adaptive clustering technique that can increase the network lifetime by further balancing the energy consumption among sensor nodes.

(MobiCom'99), Seattle Washington, USA, August 1999, pp. 263-270.

[5] Kirk Martinez, Jane K. Hart, and Royan Ong, "Environmental Sensor Networks," IEEE Computer, vol. 37, no. 8, pp. 50-56, August 2004.

[6] Lakshman Krishnamurthy, et al., "Design and Deployment of Industrial Sensor Networks: Experiences from a Semiconductor Plant and the North Sea," Proc. 3rd international conference on Embedded networked sensor systems (ACM SenSys'05), San Diego, CA, USA, November 2005, pp. 51-63.

[7] Emil Jovanov, Aleksandar Milenkovic, Chris Otto, and Piet C. de Groen, "A wireless body area network of intelligent motion sensors for computer assisted physical rehabilitation," Journal of neuroengineering and rehabilitation, vol. 2, no. 6, March 2005.

[8] P. Juang, H. Oki, Y. Wang, M. Martonosi, Li-Shiuan Peh, and D. Rubenstein. "Energy-Efficient Computing for Wildlife Tracking: Design Tradeoffs and Early Experiences with ZebraNet," Proc. 10th International Conference on Architectural Support for Programming 
Languages and Operating Systems (ASPLOS-02), San Jose, CA, USA, October 2002, pp. 96-107.

[9] S. Tilak, N. B. Abu-Ghazaleh, and W. Heinzelman, "Taxonomy of wireless micro-sensor network models," ACM SIGMOBILE Mobile Computing and Communications Review, vol. 6, no. 2, pp. 28-36, April 2002.

[10] Quanhong Wang, H. Hassanein, and Takahara, G., "Stochastic Modeling of Distributed, Dynamic, Randomized Clustering Protocols for Wireless Sensor Networks," Proc. International Conference on Parallel Processing Workshops (ICPPW'04), Montreal, Quebec, Canada, August 2004, pp. 456-463.

[11] W. B. Heinzelman, A. P. Chandrakasan, H. Balakrishnan, "An application-specific protocol architecture for Wireless Microsensor Networks", IEEE transactions on Wireless Communications, vol. 1, no. 4, pp.660-670, Oct. 2002.

[12] W. Heinzelman, A. Chandrakasan, H. Balakrishnan, "Energy-Efficient Routing Protocols for Wireless Microsensor Networks", Proc. $33^{\text {rd }}$ Hawaii International conference on System Sciences (HICSS 2000), USA , Jan. 2000, vol. 2.

[13] A. Manjeshwar, and D. P. Agrawal, “TEEN: A Routing Protocol for Enhanced Efficiency in Wireless Sensor Networks", $15^{\text {th }}$ IEEE International Parallel and Distributed Processing Symposium (IPDPS 2002), USA, April 2002, pp. 2009-2015.

[14] S. Lindsey and C. Raghavendra, "PEGASIS: PowerEfficient Gathering in Sensor Information Systems,” Proc. IEEE Aerospace Conference, 2002, vol. 3, 9-16, pp. 112530.

[15] Tillapart, P., et al, "Method for cluster heads selection in wireless sensor networks," Proc. IEEE Aerospace Conference, Big sky, MT, USA, March 2004, vol. 6, pp. 3615 - 3623.

[16] Zanxin Xu, Jian Yuan, Zhenming Feng, “D2EEC: A Distributed Degree-Based Energy Efficient Clustering Algorithm for Wireless Sensor Networks," Proc. $2^{\text {nd }}$ IEEE/ASME International Conference on Mechatronic and Embedded Systems and Applications, Beijing, P. R. China, Aug. 2006.

[17] M. Ettus, “System capacity, latency, and power consumption in multihop-routed SS-CDMA wireless networks,"Proc. Radio and Wireless Conference (RAWCON 98), Colorado Springs, CO,USA, Aug. 1998, pp. 55-58.

[18] T. Shepard, "A channel access scheme for large dense packet radio networks," in Proc. ACM SIGCOMM, Stanford, CA, Aug. 1996, pp. 219-230.
[19] V. Mhatre et. al, "A minimum cost heterogeneous sensor network with a lifetime constraint”, IEEE Transactions on Mobile Computing, vol. 4, no. 2, pages 4 -15, Jan. 2005.

Abdul Sattar Malik received the B.S. and M.S. degrees in electrical engineering from University of Engineering \& Technology, Lahore, in 2000 and 2005 respectively. He is assistant professor at the Department of Electrical Engineering, university college of Engineering \& Technology, Bahauddin Zakariya University Multan, Pakistan. These days he is pursuing his doctoral degree at Beijing Institute of Technology. His research areas lie in the area of low-power ad-hoc wireless protocol architecture, and distributed clustering protocols for wireless sensor networks.

Prof. Dr. Jingming Kuang received the Master of Engineering degree with specialization in Information and Signal Processing major from Beijing Institute of Technology 1981 and received the $\mathrm{PhD}$ degree from communication technology lab, Department of Electricity, Berlin Technology University, Germany in May, 1988. Currently he is working as professor at Beijing Institute of Technology. From July of 1999 to August of 2007, he had been the President of Beijing Institute of Technology and the dean of graduate school.

His research interests include theory and techniques of wireless communication and digital signal processing. He has supervised a number of national scientific projects and has received many awards at the national level for his outstanding contributions.

Prof. Dr. Jiakang Liu received the Bachelor and Master degree from Beijing Institute of Technology. In 1991, he received the $\mathrm{PhD}$ degree from Modern Communication Lab, Department of Electronic Engineering, Beijing Institute of Technology. In 1996 and 2003, he went to University of Siegen in Germany and the British Queen's University respectively as a visiting scholar. These days, he is working as professor of Beijing Institute of Technology. His research interests include theory and techniques of wireless communication and communication networks.

Wang Chong received the Bachelor degree from Beijing Institute of Technology in 2004. He has been on his postgraduate and doctoral programs of study at Modern Communication Lab, Department of Electronic Engineering, Beijing Institute of Technology since 2006. His research interests include theory and techniques of wireless communication and communication networks. 